\title{
Mauriac Syndrome: A Rare Complication of Type 1 Diabetes Mellitus
}

\author{
Maria João Ferreira Pinto, Nuno Melo, Luís Flores, Francisco Cunha \\ Department of Internal Medicine, Centro Hospitalar Universitário de São João, E.P.E., Porto, Portugal
}

Doi: 10.12890/2018_000969 - European Journal of Case Reports in Internal Medicine - @ EFIM 2018

Received: 01/10/2018

Accepted: $24 / 11 / 2018$

Published: 06/12/2018

How to cite this article: Ferreira Pinto MJ, Melo N, Flores L, Cunha F. Mauriac syndrome: a rare complication of type 1 diabetes mellitus. EJCRIM 2018;5: doi:10.12890/2018_000969.

Conflicts of Interests: The Authors declare that there are no competing interests.

This article is licensed under a Commons Attribution Non-Commercial 4.0 License

\section{ABSTRACT}

Mauriac syndrome, first described in 1930, is typically diagnosed in young patients with poorly controlled type 1 diabetes mellitus and growth retardation, delayed puberty, Cushingoid features, hypercholesterolaemia and hepatomegaly. However, the sole presenting feature of Mauriac syndrome can be hepatic glycogenosis in both adults and children. The mainstay of treatment for hepatic glycogenosis is strict control of glucose levels, with an excellent prognosis with improved glycaemic control. The authors present the case of a 22 -year-old female patient with type 1 diabetes mellitus and a history of poor glycaemic control who was admitted with diabetic ketoacidosis (DKA). She complained of episodes of right upper quadrant abdominal pain associated with nausea and vomiting for the last 2 months with worsening in the last 48 hours. Physical examination was remarkable for short stature and tenderness over the hepatic area with a mildly enlarged liver. The patient had elevated liver enzymes and persistent hyperlactacidaemia despite DKA resolution. Liver imaging suggested diffuse fat infiltration. The clinical suspicion of hepatic glycogenosis was confirmed by liver biopsy. After glycaemic control was improved, liver enzymes normalized and the episodes of abdominal pain, nausea and vomiting subsided.

\section{LEARNING POINTS}

- Hepatic glycogenosis can be the sole presenting feature of Mauriac syndrome.

- Hepatic glycogenosis is an under-recognized cause of abdominal pain in patients with type 1 diabetes mellitus.

- Hepatic glycogenosis may be confused with non-alcoholic fatty liver disease, with important therapeutic implications and a distinct prognosis.

- The mainstay of treatment for hepatic glycogenosis is strict glycaemic control with an excellent prognosis when achieved.

\section{KEYWORDS}

Mauriac syndrome, hepatic glycogenosis, chronic liver disease, type 1 diabetes mellitus

\section{CASE DESCRIPTION}

We present the case of a 22-year-old female patient who presented in the Emergency Department with complaints of gradually worsening episodes of right upper quadrant abdominal pain and nausea and vomiting for the past 2 months with persistent nausea and several episodes of vomiting unrelated to food intake in the last 48 hours. She denied fever or diarrhoea, had no suspicious food intake over the last few days, and had no apparent epidemiological context for viral gastroenteritis. Physical examination was remarkable for short stature, ketotic breath and tenderness over the hepatic area with a mildly enlarged liver, with no other relevant findings. She had no signs of delayed puberty or Cushingoid features. 
She had no family history of hepatic disease, significant alcohol intake or herbal, drug or over-the-counter medication exposure. Her past medical history included long-standing type 1 diabetes mellitus (T1DM), diagnosed when she was 14 years old, with poor metabolic control. She had multiple previous hospital admissions due to hypoglycaemia and diabetic ketoacidosis (DKA), with five admissions in the previous 3 years. Despite regular education concerning multiple injection therapy (insulin aspart and insulin detemir), blood glucose monitoring and an optimized nutritional plan, she maintained glycaemic variability with frequent hypoglycaemia (with no particular pattern) and several personal reports of hyperglycaemia (>300 mg/dl), mainly after meals. She was medicated with insulin detemir 10+7 IU and insulin aspart (four times daily, before meals), administered in the thighs and arms due to abdominal lipodystrophy, and had a $\mathrm{HgA} 1 \mathrm{c}$ of $13.9 \%$ at the last consultation. Measurement with a glucose meter confirmed the patient's records, but the attending physician continued to have doubts concerning the patient's compliance with her diet. She also had dyslipidaemia (total cholesterol $257 \mathrm{mg} / \mathrm{dl}$, LDL cholesterol $176 \mathrm{mg} / \mathrm{dl}$, triglycerides $240 \mathrm{mg} / \mathrm{dL}$ at diagnosis 5 years previously), with response to a high-potency statin although above the LDL recommended cutoff (LDL $96 \mathrm{mg} / \mathrm{dl}$ on rosuvastatin $20 \mathrm{mg}$ daily).

\section{METHODS AND PROCEDURES}

The diagnosis of diabetic ketoacidosis was made in the Emergency Department: laboratory results showed metabolic acidosis with a $\triangle$ anion gap $/ \Delta \mathrm{HCO}_{3}$ ratio of 1.4 , glucose level of $198 \mathrm{mg} / \mathrm{dl}$, serum lactate of $6.87 \mathrm{mmol} / \mathrm{dl}$ and ketonuria. Renal function was preserved, with mild hyperkalaemia $(5.5 \mathrm{mEq} / \mathrm{l})$, with no other significant electrolyte disturbances. She began the institution's DKA protocol and was admitted to an intermediate care unit. Additional laboratory data revealed features of hepatitis with an elevated ALT (389 U/I), AST (194 U/I) and AF $(128 \mathrm{U} / \mathrm{I})$. Coagulation, total bilirubin, serum iron and ferritin were normal. An autoimmune panel was negative. Ultrasound imaging showed hepatomegaly with uniform echogenicity, suggesting diffuse hepatic steatosis, the gallbladder had no calculi and intra-hepatic biliary ducts showed no ectasia. At this time, the differential diagnosis was between non-alcoholic fatty liver disease and hepatic glycogenosis (HG). However, a liver biopsy showing extensive clearing of the cytoplasm due to glycogen deposition and no steatosis or signs of fibrotic scaring, confirmed the diagnosis of HG (Fig. 1).

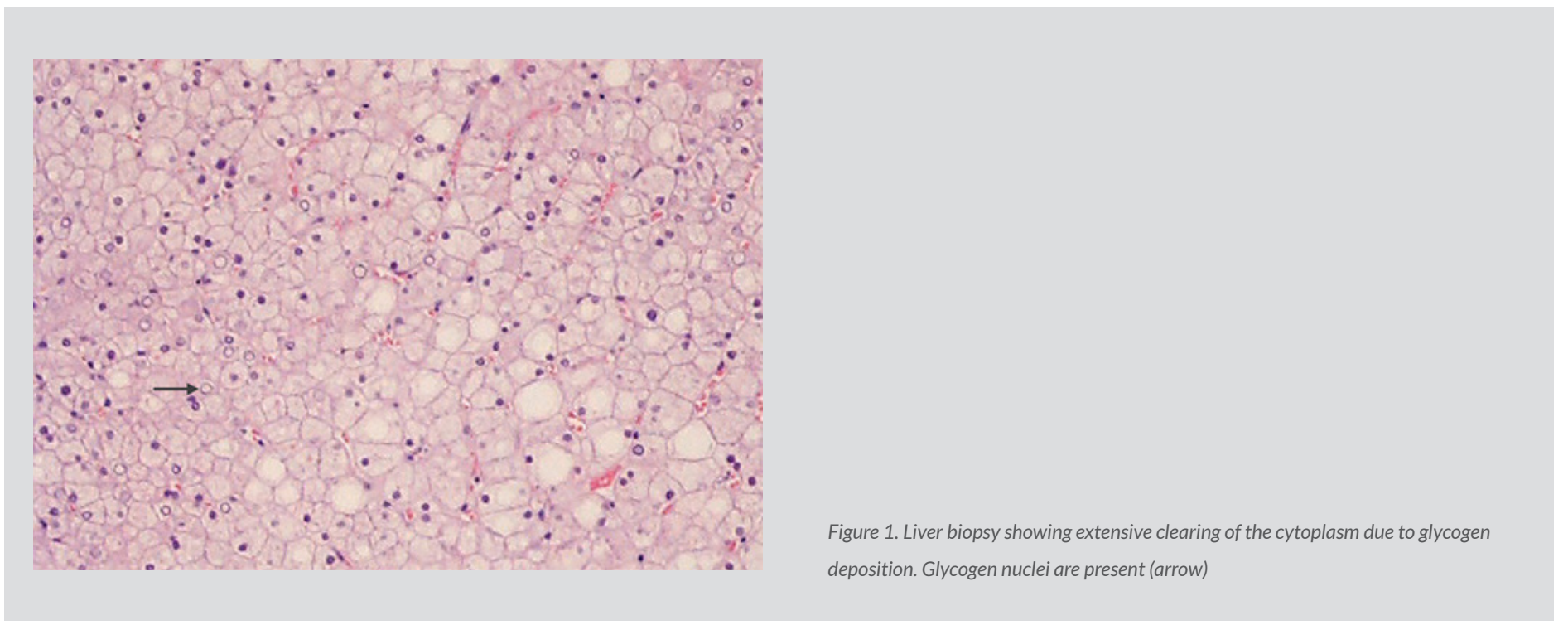

Two months after the diagnosis, the patient was started on insulin pump therapy, increased her normal calorie intake and received health and lifestyle behaviour coaching. After starting insulin pump therapy she had no more hospital admissions for DKA or other acute diabetes complications, and her glycaemic control improved considerably (HbA1C 7.9\% after 6 months). Nearly 12 months after the index hospital admission, abdominal pain had subsided and liver enzymes had normalized.

\section{DISCUSSION}

Glycogen accumulation in the liver was first described by Mauriac in 1930 as a component of Mauriac syndrome in T1DM children with poor diabetic control, hepatomegaly and abnormal liver enzymes, glycogen accumulation in the liver as well as other features such as growth retardation, delayed puberty, Cushingoid features and hypercholesterolemia ${ }^{[1]}$. 
Hepatic glycogenosis can be the sole presenting feature of Mauriac syndrome in both adults and children, develops due to excessive glycogen accumulation in hepatocytes, and is characterized by hepatomegaly and elevated liver transaminases ${ }^{[2]}$.

Although the pathogenesis has not been fully elucidated, the synthesis of hepatic glycogen seems to be a consequence of the combination of high blood glucose levels (which promote the flow of glucose into hepatocytes) and hyperinsulinaemia (which stimulates the conversion of glucose to glycogen). Hepatic glycogenosis has also been reported in patients with insulin-dependent type 2 diabetes, in high-dose steroid therapy, in dumping syndrome after gastric bypass, and in anorexia nervosa, where the combination of high blood glucose and hyperinsulinaemia is also present ${ }^{[3]}$. The clinical presentation of hepatic glycogenosis is not specific and so suspicion should be raised in the presence of abdominal right upper quadrant discomfort, hepatomegaly and abnormal liver function tests ${ }^{[2]}$.

The true incidence and prevalence of Mauriac syndrome is unknown. This may be due to the fact that most cases are never reported. Other cases of hepatic glycogenosis as the main feature of Mauriac syndrome may be incorrectly labelled non-alcoholic fatty liver disease (NAFLD) which condition cannot be distinguished from HG by history, physical examination or laboratory blood tests, with important therapeutic implications and a distinct prognosis.

Patients with Mauriac syndrome are still seen despite the global improvement in metabolic control in patients with T1DM. When encountering patients with T1DM who present with liver dysfunction, hepatomegaly and fatty liver, clinicians should consider the diagnosis of Mauriac syndrome and a liver biopsy should be performed. Treatment of Mauriac syndrome and glycogenic hepatopathy involves improved blood glucose management. Resolution of symptoms, normalization of liver enzymes and resolution of hepatomegaly has been demonstrated in our patient. Continuous insulin delivery and continuous glucose measurement might be an appropriate approach for treating these patients in order to improve clinical outcome.

\section{REFERENCES}

1. Giordano S, Martocchia A, Toussan L, et al. Diagnosis of hepatic glycogenosis in poorly controlled type 1 diabetes mellitus. World J Diabetes 2014;5:882-888.

2. Julián MT, Alonso N, Ojanguren I, Pizarro E, Ballestar E, Puig-Domingo M. Hepatic glycogenosis: an underdiagnosed complication of diabetes mellitus? World J Diabetes 2015;6:321-325.

3. Sherigar JM, Castro J, Yin YM, Guss D, Mohanty SR. Glycogenic hepatopathy: a narrative review. World J Hepatol 2018;10:172-185. 